\title{
UTILIZING UNDERWATER THREE-DIMENSIONAL MODELING TO ENHANCE ECOLOGICAL AND BIOLOGICAL STUDIES OF CORAL REEFS
}

\author{
J. H. R. Burns ${ }^{\text {a, }}{ }^{*}$, D. Delparte ${ }^{\text {b }}$, R. D. Gates ${ }^{\text {c }}$, M. Takabayashi ${ }^{\text {d }}$ \\ ${ }^{a}$ Department of Biology, College of Natural Sciences, University of Hawai‘i at Mānoa, 2538 McCarthy Mall, Edmondson Hall \\ Room 216, Honolulu, HI 96822, USA - johnhr@hawaii.edu \\ ${ }^{\mathrm{b}}$ Department of Geosciences, Idaho State University, 921 S. 8th Ave., STOP 8072. Pocatello, ID 83209, USA - delparte@isu.edu \\ ${ }^{\mathrm{c}}$ Hawai'i Institute of Marine Biology, University of Hawai'i at Mānoa, PO Box 1346, Kaneohe, HI 96722, USA - \\ rgates@hawaii.edu \\ ${ }^{\text {d}}$ Marine Science Department, University of Hawai‘i at Hilo, 200 W. Kawili St., Hilo, HI 96720, USA - misakita@hawaii.edu
}

\section{Commission V}

KEY WORDS: Coral Reefs, Coral Ecology, Habitat, Photogrammetry, Structure-from-Motion, Structural Complexity, 3D topographic reconstruction, 3D underwater modeling

\begin{abstract}
:
The structural complexity of coral reefs profoundly affects the biodiversity, productivity, and overall functionality of reef ecosystems. Conventional survey techniques utilize 2-dimensional metrics that are inadequate for accurately capturing and quantifying the intricate structural complexity of scleractinian corals. A 3-dimensional (3D) approach improves the capacity to accurately measure architectural complexity, topography, rugosity, volume, and other structural characteristics that play a significant role in habitat facilitation and ecosystem processes. This study utilized Structure-from-Motion (SfM) photogrammetry techniques to create 3D mesh models for several Hawaiian corals that represent distinct morphological phenotypes. The orthophotos and digital elevation models generated from the SfM process were imported into geospatial analysis software in order to quantify several metrics pertaining to 3D complexity that are known to affect ecosystem biodiversity and productivity. The 3D structural properties of the reconstructed coral colonies were statistically analyzed to determine if the each species represents a unique morpho-functional group. The SfM reconstruction techniques described in this paper can be utilized for an array of research purposes to improve our understanding of how changes in coral composition affect habitat structure and ecological processes in coral reef ecosystems.
\end{abstract}

\section{INTRODUCTION}

Reef forming corals provide the structural foundation of highly diverse and productive coral reef ecosystems. Coral reefs are among the most ecologically and economically productive ecosystems on the planet, supporting an estimated eukaryotic species richness ranging from 650,000 to over 9 million (ReakaKudla 1997), and generating an estimated US\$375 billion each year through recreation, tourism, waste treatment, and production of raw materials (Costanza et al. 1997, HoeghGuldberg et al. 2007). Coral reefs have experienced severe declines in integrity due to increased intensity and frequency of environmental stress associated with global climate change and local anthropogenic disturbances (Harvell et al. 1999, HoeghGuldberg et al. 2007). Loss of corals detrimentally affects an array of ecological processes and can result in phase shifts from coral to algal dominated states. Degradation and recovery of corals are typically measured through two-dimensional (2D) assessments of coral cover; however, this metric fails to capture changes occurring in architectural complexity (Alvarez-Filip et al. 2011). Precisely quantifying the structural characteristics of corals is essential for assessing how mortality or shifts in composition affect critical ecosystem services.

The importance of the three-dimensional (3D) structure and topographic complexity of coral reefs to the ecological function of these ecosystems has been recognized for a number of decades (Risk 1972, Luckhurst and Luckhurst 1978). Structural complexity in ecosystems creates an assortment of microhabitat types and has been shown to support greater diversity and abundance of associated organisms (MacArthur and MacArthur 1961, Crowder and Cooper 1982, Graham and Nash 2013). In an ecological context, it has been suggested that the two primary variables driving coral reef resilience are reef framework and reef-building capacity, both of which can be quantified with 3D measures of topographic structure (Done 1997, Fisher et al. 2007). The increase in disturbance and degradation of these environments has made accurate quantification of structural complexity increasingly important. Unfortunately, the cost and technical difficulty of collecting accurate $3 \mathrm{D}$ information from underwater environments has limited the ability to decipher specific structural elements responsible for driving ecological processes. Developing a feasible and accurate approach for 3D reconstruction will allow for determining how disturbance, mortality, and phase shifts affect the biodiversity and productivity of coral reef ecosystems.

Creating 3D reconstructions of natural environments has historically been a challenging and costly undertaking. However, advancements in both computer technologies and computer vision algorithms now make it possible to efficiently reconstruct the 3D geometry of fixed objects. Structure-fromMotion is a recently developed photogrammetric technology that provides a simple and low-cost method for high-resolution 3D topographic reconstruction. SfM determines the geometry of the photographed scene, camera positions and geometry without the need for a network of targets with known 3D positions (Snavely et al. 2008, Westoby et al. 2012). Camera pose and 3D geometry are reconstructed simultaneously by automatically matching invariant features among overlapping images (Snavely et al. 2008, Westoby et al. 2012). The features are tracked throughout the image processing to develop initial estimates of object coordinates, which are then further refined with highly redundant bundle adjustments (Snavely et al. 2008, Westoby et 
al. 2012). The end result of the process is a dense 3D point cloud of identifiable features in a relative coordinate system. Once georeferenced, this point cloud can be used to quantify 3D characteristics of the reconstructed scene (Fonstad et al. 2013). Automating the process of detecting invariant features to 3D reconstruction of scene geometry makes SfM more efficient and cost-effective than traditional photogrammetric methodologies. Several studies have validated the accuracy of SfM products for high-resolution 3D topographic analyses, and in some cases found SfM to be comparable to more expensive LIDAR techniques (Delparte et al. 2014, Javernick et al. 2014).

The SfM technology is capable of producing multiple 3D products that can be analyzed with terrain analysis tools. Digital elevation models (DEMs) can be exported into geospatial software in order to quantify $3 \mathrm{D}$ topographical features that are relevant to ecosystem function. This process enables quantification of intricate $3 \mathrm{D}$ features such as surface complexity (3D/2D surface area), slope, volume and curvature. These metrics are known to be important predictors of organismal abundance, biomass and diversity, and also affect benthic current velocities associated with the food particle supply for suspension feeding corals (Kostylev et al. 2005, Guinan et al. 2009, Pittman et al. 2009, Pittman and Brown 2011). The ability to characterize these topographic features will greatly enhance both biological and ecological investigations of coral reef ecosystems.

This objective of this study was to develop a process for combining 3D data derived from SfM photogrammetry with geospatial software tools in order to quantify $3 \mathrm{D}$ topographic characteristics of individual coral colonies. This technique enables the extraction and quantification of 3D structural features that are biologically and ecologically relevant to coral reef ecosystems. We utilized this approach to compare four dominant morphologies exhibited by four species of Hawaiian corals. The approach presented here can be applied to larger studies in order to distinguish key morpho-functional groups of corals that promote architectural complexity and drive ecological processes in reef ecosystems.

\section{METHODS}

\subsection{Photographing coral colonies}

Images were collected at Wai'ōpae tide pools $\left(19^{\circ} 29^{\prime} 55^{\prime \prime} \mathrm{N}\right.$ $154^{\circ} 49^{\prime} 06^{\prime \prime}$ W), southeast Hawai'i Island. Several ground control points (GCPs) and scale markers were placed around each photographed colony at known spatial locations and depths to accurately georeference and ground-truth the resulting models. Photographs were taken continuously from both planar and oblique angles to collect images that captured the entire colony surface area with an overlap of $70-80 \%$. All photos were taken using a Canon 5D Mark III digital SLR camera with a $14 \mathrm{~mm}$ non-distortion lens (Canon USA Inc.) in an Ikelite housing (Ikelite Underwater Systems). Three colonies of each species (a total of twelve colonies) were photographed, each representing a unique morphology (Porites lobata massive/lobate, Porites compressa - branching, Montipora capitata - plating, Montipora flabellata - encrusting).

\subsection{D reconstructions}

3D colony reconstructions were rendered using Agisoft PhotoScan (Agisoft LLC.) modeling software. All images were uploaded into the software, and PhotoScan performed camera calibration using Brown's distortion model with focal information derived from the camera metadata. Once the photos were uploaded the software aligned the images using algorithms capable of detecting invariant features that overlap among the sequential images. The invariant features were used to develop geometrical projective matrices and determine the position and orientation of the camera for each corresponding image (Verhoeven et al. 2012; Westoby et al. 2012). 3D geometry was constructed on the 2D image plan using the extrinsic parameters calculated during the photo alignment process in conjunction with the intrinsic parameters and focal properties obtained from the image metadata (Stal et al. 2012). The photo alignment process used iterative bundle adjustments to create a sparse 3D point cloud that represents the projection and intersection of pixel rays from the different positions and orientation of the images in a 3D space. Digital markers were manually annotated onto all of the GCPs, and the location of each marker was reviewed and corrected in all photos containing the GCPs. The known $\mathrm{x}, \mathrm{y}, \mathrm{z}$ values for each GCP were inputted into the software to optimize alignment of the images and ensure accurate interior and exterior orientation of the resulting model. Once the photo alignment was optimized a dense point cloud could was generated. The dense point was used to build a continuous mesh surface, which was triangulated and rendered with the high-resolution imagery to create a textured digital surface model for each colony.
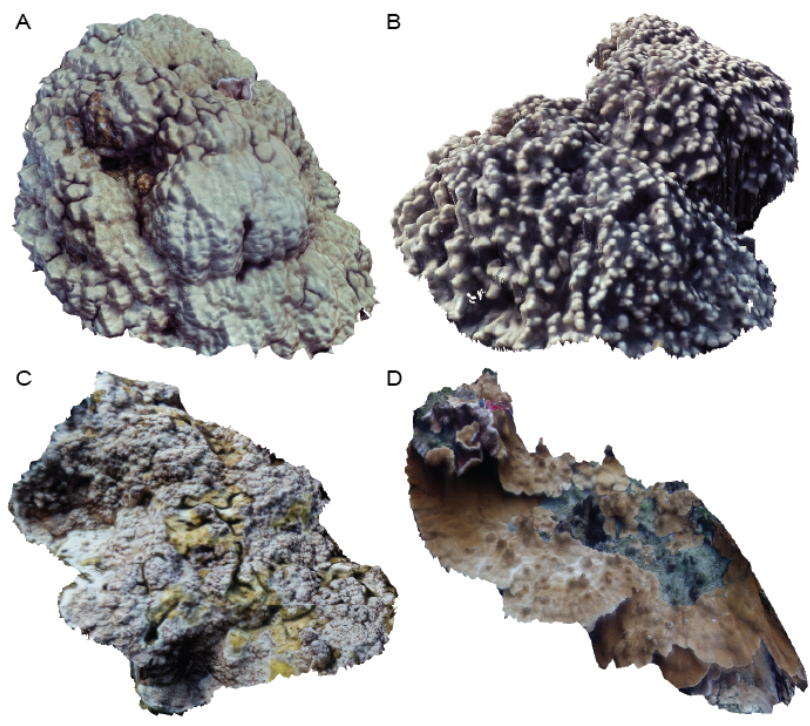

Figure 1. Example 3D models of the four coral morphologies analyzed in this study; a) massive - Porites lobata, b) branching - Porites compressa, c) encrusting - Montipora flabellata, d) plating-Montipora capitata.

\subsection{Quantification of 3D features}

3D characteristics were quantified using 3D spatial analysis tools in ArcGIS (ArcMap 10.1, Environmental Systems Resource Institute) topographic software. Both an orthophoto and a digital elevation model (DEM) were exported from Photoscan and uploaded into ArcMap for each reconstructed coral colony. The DEM cell size was set to $0.1-\mathrm{cm}$ so intricate differences in 3D features could be detected among the colonies. The orthophoto was layered on top of the DEM, and each colony was digitized using the editor function within ArcMap. Once annotated, 3D metrics were computed using several 3D spatial analysis tools. 'Surface rugosity' was quantified as the contour distance (accounting for changes in 
vertical surfaces along the colony surface) divided by the linear distance between two nodes. The path distance tool was used to quantify a mean surface rugosity value from four rugosity planes that were digitized on each coral colony (twelve total for each species). The surface information tool was used to quantify the 2D and 3D surface area for each coral colony. A mean ratio of $3 \mathrm{D} / 2 \mathrm{D}$ surface area was calculated for each colony as a representation of 'surface complexity'. The slope tool was used to determine the maximum rate of elevational change from each cell to its neighbors, in a $3 \times 3$ cell window, in units of degrees. The value of the slope plane is calculated with the average maximum technique, with small values reflecting flat terrain and large values indicating steep terrain (Burrough and McDonell 1998). The values were computed as percent slope, which is tangent of $\theta$ (rise/run) multiplied by 100 . A slope angle of 45 degrees, with the rise equal to run, has a percent slope value of 100 and as the angle approaches vertical the percent slope value approaches infinity. The curvature tool was used to quantify the second derivative of slope across the DEM surface in order to identify areas of rapid change in slope or aspect. The curvature of the surface is calculated on a cell-by-cell basis for the entire DEM. For each cell, a fourth-order polynomial of the form:

$$
Z=A x^{2} y^{2}+B x^{2} y+C x y^{2}+D x^{2}+E y^{2}+F x y+G x+H y+I
$$

is fit to a surface composed of a $3 \times 3$ cell window. Values were quantified for combined curvature, planform curvature, and profile curvature. Combined curvature provides a value that is representative of all directional changes in slope or aspect within the analyzed cell windows, thus large values of combined curvature are indicative of complex terrain. Profile curvature only analyzes changes in slope or aspect parallel to the direction of maximum slope, while planform curvature analyzes changes in the perpendicular direction of maximum slope. The positive or negative values of planform and profile curvature represent either upwardly concave or convex surface (Figure 2).

\subsection{Statistical Analysis}

Data generated from the topographic analyses were transformed, if necessary, using log transformations, to meet the assumptions of normality and equal variance necessary for use of parametric statistical tests. Values for the spatial metrics described above were analyzed using univariate analysis of variance (ANOVA), and Tukey's HSD post hoc tests were used to determine statistical differences $(\alpha=0.05)$ among the quantified spatial metrics. All statistical tests were run using Minitab 15 (Minitab Inc.) software.

\section{RESULTS}

The ground sample distance (resolution/pixel) of the resulting $3 \mathrm{D}$ models ranged from $0.000310-0.000344-\mathrm{m} / \mathrm{pix}$ with an error of $1.74-2.98$ pix, thus our designated DEM cell size of $0.1-\mathrm{cm}$ was within the range of accuracy of the model. $100 \%$ of the images were matched for each colony reconstruction.

Surface rugosity differed significantly among the coral colonies (ANOVA, $\mathrm{F}=4.48, \mathrm{p}<0.01$, Figure 2), with the massive morphology $(P$. lobata) exhibiting greater mean values of rugosity than the encrusting morphology ( $M$. flabellata). Surface complexity was significantly greater (ANOVA, $\mathrm{F}=$ $18.11, \mathrm{p}<0.01$, Figure 2 ) in the corals exhibiting massive and branching morphologies ( $P$. lobata and $P$. compressa) compared to the encrusting and plating morphologies $(M$. capitata and M. flabellata). Percent slope was also significantly greater (ANOVA, $\mathrm{F}=24.49, \mathrm{p}<0.01$, Figure 2 ) in the corals exhibiting massive and branching morphologies ( $P$. lobata and $P$. compressa) compared to the encrusting and plating morphologies (M. capitata and M. flabellata).

The mean values of combined, profile, and planform curvature varied among the coral morphologies (Figure 2). P. lobata and $P$. compressa, coral species with branching and massive morphologies, exhibited greater values of both planform and profile curvature.

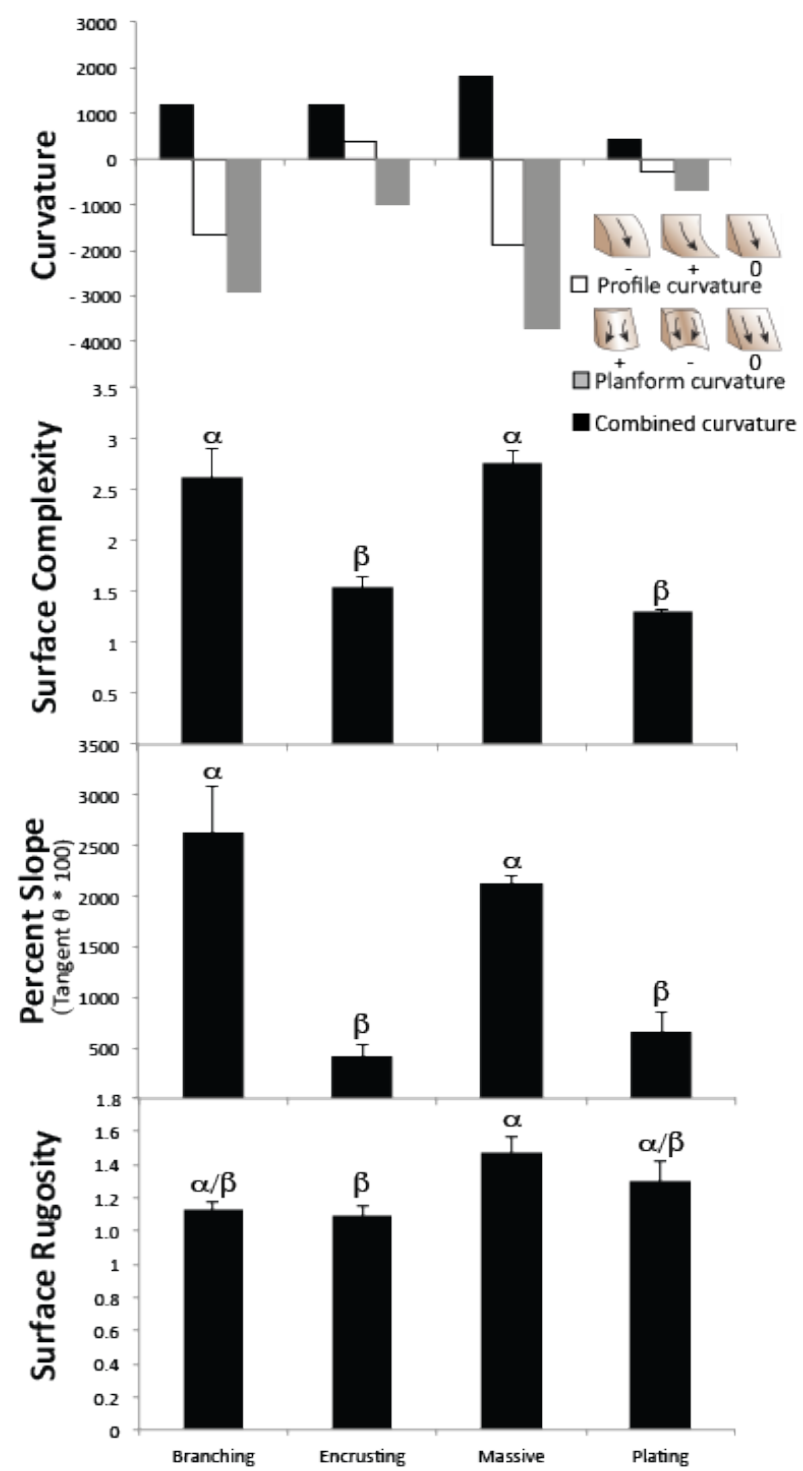

Figure 2. Statistical comparisons among mean values of 3D metrics. . $\alpha, \beta$, and $\gamma$ demark groupings identified as significantly different based on statistical analyses (ANOVA, $\mathrm{p}<0.01)$.

\section{CONCLUSIONS}

The field of coral reef ecology has recognized complexity to be a critical component of ecosystem diversity and productivity (Goreau 1959, Knudby and LeDrew 2007, Walker et al. 2009, Dustan et al. 2013), yet there are few methods capable of quantifying intricate $3 \mathrm{D}$ features of underwater environments. 
Obtaining accurate 3D measurements of coral surfaces has only been limited to techniques using complex, bulky, and expensive equipment, thus highlighting a clear need for non-invasive methods to measure 3D surface area and complexity for corals in situ (Bythell et al. 2001). This study describes the application of a novel technique for combining $3 \mathrm{D}$ products generated from Structure for Motion (SfM) photogrammetry with geospatial software tools for characterizing 3D attributes of coral colonies. The methods presented here provide a cost- and time-effective approach to reconstruct coral colonies and quantify intricate 3D features associated with specific coral morphologies.

Our aim was to develop a process to quantify $3 \mathrm{D}$ metrics that are known to affect habitat complexity and ecosystem processes. Surface rugosity, surface complexity, slope, and curvature have been shown to drive vegetation distribution on terrestrial landscapes and influence the distribution, biomass and diversity of vertebrate and invertebrate species in marine systems (Friedlander and Parrish 1998, Kostylev et al. 2005, Guinan et al. 2009, Pittman et al. 2009, Pittman and Brown 2011). We quantified these metrics for four coral species found commonly in Hawai' $i$ that represent distinct morphological phenotypes (Porites lobata - massive/lobate, Porites compressa - branching, Montipora capitata - plating, Montipora flabellate - encrusting). The resulting data showed the branching and massive corals to exhibit the greatest levels of surface rugosity, surface complexity, and slope (Figure 2). These morphologies also exhibited larger values of profile and planform curvature, and thus are likely to provide more complex habitat than the plating and encrusting morphologies. The negative values of both profile and planform curvature indicate the coral surface provides a juxtaposition of both concave and convex shape, which may facilitate more functional habitat for organisms that use coral as shelter. The architectural complexity of ecosystems often drives ecological services, and is frequently defined or provided by foundation taxa that disproportionately influence ecosystem structure, function and stability (MacArthur 1984, Bruno and Bertness 2001, Alvarex-Filip et al. 2011). Corals serve as foundation taxa for coral reef ecosystems, and different species can disproportionately contribute to architectural complexity (Alvarez-Filip et al. 2011). Unfortunately, there are few studies that have directly distinguished, or comprehensively examined, morpho-functional groups and their role in habitat facilitation on coral reefs. It is important to identify the structural features associated with specific coral species and morphologies in order to determine if distinct morphofunctional groups exist, and to what degree they drive ecosystem diversity and productivity. Understanding the intricate differences in the architectural complexity provided by morpho-functional groups of corals can also elucidate how shifts in coral composition may affect ecosystem structure and function. Quantifying the relative contribution of specific coral species and morphologies to the architectural complexity of coral reefs is of particular importance for understanding the trajectory of coral reefs under changing environmental conditions (Alvarez-Filip et al. 2011).

Developing 3D reconstructions of corals can also serve as a valuable tool for tracking growth rates and assessing the impacts of short-term environmental perturbations. The complex branching, plating and corymbose coral morphologies have been found to be generally more susceptible to disturbance than slow-growing corals that exhibit massive morphologies (Gates and Ainsworth 2011, van Woesik et al. 2011). Utilizing the technique presented here can enable comparison of $3 \mathrm{D}$ growth rates over time, and also assess structural changes occurring in relation to disturbance events such as coral bleaching, disease outbreaks, and severe storms.

Combining 3D products derived from SfM photogrammetry with geospatial analysis tools has wide applications in coral reef research and management. Furthermore, this method provides high-resolution visual tools for assessing changes over time, such as the orthophoto and photo-mosaic, and the point clouds can be compared across temporal scales in order to precisely quantify changes occurring in the volumetric and structural properties of corals. This technique is critical for accurately investigating parameters such as coral biomass and habitat availability for reef fishes and invertebrates, all of which plays a vital role in coral reef productivity (Luckhurst and Luckhurst 1978, Abdo et al. 2006, Wilson et al 2007). We hope the methodology presented in this study can serve as a platform for future studies to accurately quantify intricate $3 \mathrm{D}$ characteristics of corals and coral reefs to improve our collective understanding of these valuable ecosystems.

\section{ACKNOWLEDGEMENTS}

This research was funded by the National Science Foundation Center for Research Excellence in Science and Technology Grant No. 0833211 to MT for the Center in Tropical Ecology and Evolution in Marine and Terrestrial Environments. This publication was developed under STAR Fellowship Assistance Agreement no. FP-91768001-0 awarded by the U.S. Environmental Protection Agency (EPA). It has not been formally reviewed by the EPA. The views expressed in this publication are solely those of the authors and EPA does not endorse any products or commercial services mentioned in this publication. Funding was also provided by both ESPCoR Hawai'i (EPS-0903833) and Idaho (EPS-0814387) Track-I research grants, and the UH Manoa Edmonson Grant. We thank the University of Hawai'i at Hilo Spatial Data Analysis and Visualization (SDAV) laboratory, and the Idaho National Laboratories for logistical support and funding involved with fieldwork and image analysis. We also thank Kanoe Steward for assistance with fieldwork.

\section{REFERENCES}

Abdo, D.A., J.W. Seager, E.S. Harvey, J.I. McDonald, G.A. Kendrick, and M.R. Shortis., 2006. Efficiently measuring complex sessile epibenthic organisms using novel photogrammetric technique. Journal of Experimental Marine Biology and Ecology 339:120-133

Alvarez-Filip, L., N.K. Dulvy, I.M. Cote, A.R. Watkinson, and J.A. Gill., 2011. Coral identify underpins architectural complexity on Caribbean reefs. Ecological Applications 21(6):2223-2231

Bruno, J.F., and M. D. Bertness., 2001. Habitat modification and facilitation in benthic marine communities. Pages 201-218 in M. D. Bertness, S. D. Gaines, and M.E. Hay, editors. Marine community ecology. Sinauer, Sunderland, Massachusetts, USA.

Burrough, P.A., and R.A. McDonell., 1998. Principles of geographical information systems. Oxford Unversity Press, New York. 190 pp

Bythell, J.C., P. Pan, and J. Lee., 2001. Three-dimensional morphometric measurements of reef corals using underwater photogrammetry techniques. Coral Reefs 20:193-199 
Costanza, R., R. d'Arge, R. de Groot, S. Farber, M. Grasso, B. Hannon, K. Limburg, S. Naeem, R.V. O’Neill, J. Paruelo, R.G. Raskin, P. Sutton, and M. van den Belt., 1997. The value of the world's ecosystem services and natural capital. Nature 387: 253260

Crowder, L.B., W.E. Cooper., 1982. Habitat structural complexity and the interaction between bluegills and their prey. Ecology 63:1802-1813

Delparte, D.M., M. Belt, C. Nishioka, N. Turner, R. Richardson, and T. Ericksen., 2014. Monitoring tropical alpine lake levels in a culturally sensitive environment utilizing 3D technological approaches. Arctic, Antarctic, and Alpine Research 46(4):709718

Done, T.J., 1997. Decadel changes in reef-building communities: implications for reef growth and monitoring programs. Proceedings of the $8^{\text {th }}$ International Coral Reef Symposium 1:411-416

Dustan, P., O. Doherty, S. Pardede., 2013. Digital reef rugosity estimates coral reef habitat complexity. PLoS ONE 8(2):e57386

Fisher, W.S., W.P. Davis, R.L. Quarles, J. Patrick, J.G. Campbell, P.S. Harris, B.L. Hemmer, and M. Parsons., 2007. Characterizing coral condition using estimates of threedimensional colony surface area. Environmental Monitoring and Assessment 125:347-360

Fonstad, M.A., J.T. Dietrich, B.C. Courville, J.L. Jensen, and P.E. Carbonneau., 2013. Topographic structure from motion: a new development in photogrammetric measurement. Earth Surface Processes and Landforms DOI:10.1002/esp.3366

Friedlander, A.M., and J.D. Parrish., 1998. Habitat characteristics affecting fish assemblages on a Hawaiian coral reef. Journal of Experimental Marine Biology and Ecology 224:1-30

Gates, R.D., and T.D. Ainsworth., 2011. The nature and taxonomic composition of coral symbiomes as drivers of performance limits in scleractinian corals. Journal of Experimental Marine Biology and Ecology 408:94-101

Goreau, T.F. 1959., The ecology of Jamaican coral reefs 1. Species composition and zonation. Ecology 40:67-90

Graham, N.A.J., and K.L. Nash., 2013. The importance of structural complexity in coral reef ecosystems. Coral Reefs $32: 315-326$

Guinan, J., C. Brown, M.F.J. Dolan, and A.J. Grehan., 2009. Ecological niche modeling of the distribution of cold-water coral habitat using underwater remote sensing data. Ecological Informatics 4:83-92

Harvell, C.D., K. Kim, J.M. Burkholder, R.R. Colwell, P.R. Epstein, D.J. Grimes, E.E. Hofmann, E.K. Lipp, A.D. Osterhaus, R.M. Overstreet, J.W. Porter, G.W. Smith, and G.R. Vasta., 1999. Emerging Marine Diseases, Climate Links and Anthropogenic Factors. Science 285:1505-1510

Hoegh-Guldberg, O., P.J. Mumby, A.J. Hooten, R.S. Steneck, P. Greenfield, E. Gomez, C.D. Harvell, P.F. Sale, A.J. Edwards, K. Caldeira, N. Knowlton, C.M. Eakin, R. Iglesias-Prieto, N. Muthiga, R.H. Bradbury, A. Dubi, and M.E. Hatziolos., 2007.
Coral Reefs Under Rapid Climate Change and Ocean Acidification. Science 318:1737-1742

Javernick, L., J. Brasington, and B. Caruso., 2014. Modeling the topography of shallow braided rivers using Structure-fromMotion photogrammetry. Geomorphology 213:166-182

Knudby, A., and E. LeDrew., 2007. Measuring structural complexity on coral reefs. In: Pollock NW, Godfrey JM, eds. Diving for Science 2007. Proc of the American Academy of Underwater Sciences $26^{\text {th }}$ Symposium pgs: $181-188$

Kostylev, V.E., J. Erlandsson, M.Y. Ming, and G.A. Williams., 2005. The relative importance of habitat complexity and surface area in assessing biodiversity: Fractal application on rocky shores. Ecological Complexity 2:272-286

Luckhurst, B.E., and K. Luckhurst., 1978. Analysis of the influence of substrate variables on coral reef fish communities. Marine Biology 49:317-323

MacArthur, R. H., 1984. Geographical ecology, patterns in the distributions of species. Princeton University Press, Princeton, New Jersey, USA.

Pittman, S.J., B.M. Costa, and T.A. Battista., 2009. Using lidar bathymetry and boosted regression trees to predict the diversity and abundance of fish and corals. Journal of Coastal Research 25(6):27-38

Pittman, S.J., and K.A. Brown., 2011. Multi-scale approach for predicting fish species distributions across coral reef seascapes. PLoS ONE 6(5):e20583

Reaka-Kudla, M., 1997. The global biodiversity of coral reefs: a comparison with rain forests. In: Reaka-Kudla M, Wilson DE, Wilson EO (eds.), Biodiversity II: Understanding and Protecting our Biological Resources. Washington, D.C.: Joseph Henry Press. pp. 83-108.

Risk, M.J., 1972. Fish diversity on a coral reef in the Virgin Islands. Atoll Research Bulletin 153

Singh, H., G. Salgian, R. Eustice, and R. Mandelbaum., 2002. Sensor fusion of structure-from-motion, bathymetric 3D, and beacon-based navigation modalities. Proceedings of the IEEE International Conference on Robotics and Automation 4:40244031

Snavely, N., S.N. Seitz, and R. Szeliski., 2008. Modeling the world from internet photo collections. International Journal of Computer Vision 80:189-210

Stal, C., J. Bourgeois, P. De Maeyer, G. De Mulder, A. De Wulf, R. Goossens, M. Hendrickx, T. Nuttens, and B. Stichelbaut., 2012. Test case on the quality analysis of structure from motion in airborne applications. Proceedings of the $32^{\text {nd }}$ EARSeL Symposium: 'Advances in Geosciences,' Mykonos, Greece, May 21-24, P.11

Van Woesik, R., K. Sakai, A. Ganase, and Y. Loya., 2011. Revisiting the winners and losers a decade after coral bleaching. Marine Ecology Progress Series 434:67-76

Verhoeven, G., 2012. Getting computer vision airborne: using structure from motion for accurate orthophoto production. RSPSOC Archaeology Special Interest Group Newsletter, 4-6 
Walker, B.K., L.K.B Jordan, and R.E. Spieler., 2009.

Relationship of reef fish assemblages and topographic complexity on Southeastern Florida Coral Reef Habitats. Journal of Coastal Research 25(6):39-48

Westoby, M.J., J. Brasington, N.F. Glasser, M.J. Hambrey, and J.M. Reynolds., $2012 . \quad$ 'Structure-from-Motion' photogrammetry: A low-cost, effective tool for geoscience applications. Geomorphology 179:300-314

Wilson, S.K., N.A.J. Graham, and N.V.C. Polunin., 2007. Appraisal of visual assessments of habitat complexity and benthic composition on coral reefs. Marine Biology 151:10691076 